\title{
Detection of lung Cancer on CT Scan Using Image Processing Techniques
}

\author{
Osman Mudathir ${ }^{1}$, Alaa Elfadel ${ }^{1}{ }^{*}$, Suha Salah ${ }^{1}$, Marwa Gamar $^{1}$ and Zeinab Nour-Aldaem ${ }^{1}$ \\ 1 Electrical Engineering Department, Omdurman Islamic University, Omdurman, Sudan \\ * Corresponding author: Alaa Elfadel (e-mail: lolaelfadel@gmail.com).
}

Article history: Received 14 July 2020, Received in revised form 04 Dec. 2020, Accepted 04 Dec. 2020

\begin{abstract}
This paper represents detection of lung cancer using image processing which is followed by image enhancement using three filters. These filters are Gabor, madian and mean filters. Then, image segmentation is applied using a technique called marker controlled watershed with masking that has advantages over other methods in terms of reducing the time needed for detection. On that ground, this method rejoiced with better quality. Finally, an important stage is made to decide whether the lung is infected with cancer or not this stage is called feature extraction therefore, results were reached with less human efforts.
\end{abstract}

Keywords: Computed Tomography (CT), Cancer, Image processing, Marker-controlled Segmentation.

\section{INTRODUCTION}

Lung cancer is a condition that causes cells to divide in the lungs uncontrollably [1]. This makes the patient unable to breathe properly. Lung cancer is generally divided into two main types. Depending on the shape of cancer cells, as seen under the microscope. Those types are Small cell lung cancer and Non-small lung cancer. Lung cancer has the highest death rate among all other types of cancer .Because smoking is responsible for about 90 percent of all lung cancer cases. The risk of developing lung cancer is steadily increasing, depending on the years and number of cigarettes smoked. It is one of the most serious cancers in the world, with very less survival opportunity.

After the diagnosis, the American Cancer Society's estimates for lung cancer in the United States for 2019 are About 228,150 new cases of lung cancer $(116,440$ in men and 111,710 in women),About 142,670 deaths from lung cancer (76,650 in men and 66,020 in women)[2]. so the process of early detection of the disease plays a very important and primary role to avoid serious advanced stages to reduce its percentage of distribution.

There are many methods and techniques for early lung cancer detection such as chest radiograph (x-ray), Computed Tomography (CT), magnetic resonance imaging (MRI). However, these techniques are expensive and time-consuming to give results. Therefore, there is a tremendous need for new techniques to detect cancer cells in their early stages. Image processing technology helps doctors and radiologists to Give the right diagnosis and also help to save time and pave the way for quick access to medical information.

\section{METHODOLOGY}

The operation of image processing is used to cut the images and the insert them in to the mean and madian for the preprocessing and then segment the image by using the technology of Marker-controlled watershed to divide the tumor and to know If there is a disease or not depending on the Features extracted. This operation goes through four stages, those stages are:

A.Image Acquisitions
B.Image Enhancement 
C.Image Segmentation

D.Feature Extraction

Sub as illustrated in Fig. 1:

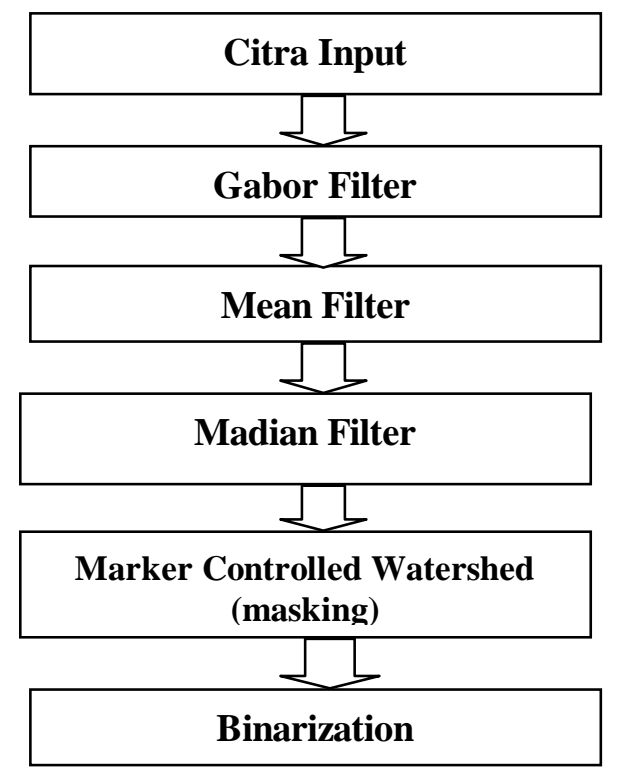

Fig.1 Block diagram of Lung Cancer Nodule Segmentation and Feature Extraction System

\section{A. Image Acquisitions:}

At this stage, a total of CT images (natural and non-natural) are taken from available database sites such as (LIDC), Physionet.org, and from hospitals. The images used in this research are taken from a hospital. The Data of the Medical images stored in DICOM format this format is a standard transport, storage [3]. the CT images has advantages over the $x$ Ray and magnetic resonance and this advantages are having Noise and disturbance less than the $x$ Ray and magnetic resonance(MRI).

\section{B. Image Enhancement:}

It is a way to improve the quality of the image in order to make the diagnosis more clear by removing noise and disturbance by using filters and modify the contrast of the image through the process of processing the initial image.

Image enhancement techniques can be divided into two broad categories: Spatial domain methods and frequency domain methods. In the image enhancement phase, three types of filters were used, they are Gabor filter, Mean filter and Madian filter.

- Gabor filter:
Gabor filter named by Dennis Gabor, A Gabor filter is a linear filter whose impulse response is defined by a harmonic function multiplied by a Gaussian function. Because of the multiplication-convolution property (Convolution theorem), the Fourier transform of a Gabor filter's impulse response is the convolution of the Fourier transform of the harmonic function and the Fourier transform of the Gaussian function [4]

$$
G(\sigma, \theta, \lambda, \psi, \gamma ; x, y)=\exp \frac{\frac{-\left(x^{2}+y^{2} y^{\prime 2}\right)}{{ }^{2} \sigma^{2}} \cdot \cos \left(2 \frac{x}{\lambda}+\psi\right)}{}
$$

- Mean filter:

This filter works to smooth the image by reducing the density between each pixel and the next.

$$
\hat{f}(x, y)=\frac{1}{m n-a} \sum_{(s, t) \in S_{x, y}} g_{r}(s, t)
$$

\section{- Madian filter:}

It reduces the salt and pepper noise from the image and often does a better job by reserving important details of images. The median is calculated by first sorting all the pixel values from the surrounding neighborhood into numerical order and then replacing the pixel being considered with the middle pixel value [5]. If the neighborhood under consideration contains an even number of pixels, the average of the two middle pixel values is used.

$$
\hat{f}(x, y)=\operatorname{median}_{(s, t) \in \in_{x y}}\{g(s, t)\}
$$

All candidates are used to remove the noise from the image, unfortunately, there is no general theory to improve the image, it is only about the perception of the human if the picture is good or not the processed image is used as an input to the next step (Segmentation).

\section{Image Segmentation:}

Image segmentation is an essential process for most image analysis subsequent tasks. The purpose of the partitioning process is to make the representation of the image simpler and easier to analyze. The watershed works to process the surface image, where the light pixels represent high altitudes and the dark pixels represent the low interactions and then divided into integer 
numbers greater than or equal to 0 The elements named 0 do not belong to a unique watershed area and the 1 elements belong to the first watershed And the so-called 2 elements of the second watershed area and so on.

The segmentation algorithms are based on two basic properties of intensity values discontinuity and similarity [6]. First category is to partition an image based on abrupt changes in intensity, such as edges in an image. Second category is based on partitioning an image into regions that are similar according to a set of predefined criteria for this paper, marker-controlled watershed with Masking segmentation In simple watershed, the problem of over segmentation is faced, To reduce this problem, marker-based watershed segmentation is used. Marker-controlled watershed with masking is an improved form of watershed. In marker-based watershed segmentation, markers are used. The marker-controlled watershed with masking segmentation has two types: External is linked to the background and internal is linked to objects, marking in areas of interest of the internal and the external works to solve the problem.

To get the result of the retail process, follow these steps:

First calculate the distance of the gradient to reveal the edges .Second recognize the desired object by using the morphological process. After the object has been detected by the marker, other areas can be ignored and focus on the area where the object is locate, then mask the object in left and right lung. There are examples of marker control watershed with masking segmentation approach that are available in the Matlab program and can be used directly.

i Region growing:

It is used to enlarge the coverage area by sub or adding pixels based on predetermined criteria.

TABLE I: DETECTION RESULT OF FIVE SAMPLE PATIENTS [7].

\begin{tabular}{lll}
\hline \hline images & $\begin{array}{l}\text { Region } \\
\text { growing }\end{array}$ & $\begin{array}{l}\text { Marker } \\
\text { controlled } \\
\text { watershed with } \\
\text { masking }\end{array}$ \\
\hline 1 & $93.259 \mathrm{~s}$ & $4.644 \mathrm{~s}$ \\
2 & $88.136 \mathrm{~s}$ & $4.639 \mathrm{~s}$ \\
3 & $87.740 \mathrm{~s}$ & $4.329 \mathrm{~s}$ \\
4 & $87.870 \mathrm{~s}$ & $4.719 \mathrm{~s}$ \\
5 & $89.316 \mathrm{~s}$ & $4.470 \mathrm{~s}$ \\
\hline \hline
\end{tabular}

ii Thresholding:

It is a non-linear operation that converts gray scale images in to binary image $(0,1)$ that are above or below a threshold value. Here Otsu approach was used .Otsu selected the minimum value related to the difference between the forefront and the background pixels and considered this value is the threshold value it's defined between 0 and 1, then the segmentation is applied based on this value .

TABLE II: IMAGE SEGMENTATION EXPERIMENTAL RESULT [8[

\begin{tabular}{lll}
\hline \hline & & $\begin{array}{l}\text { Marker } \\
\text { Threshold } \\
\text {-ing }\end{array}$ \\
& & $\begin{array}{l}\text { controlled } \\
\text { watershed } \\
\text { with } \\
\text { masking }\end{array}$ \\
\hline Sub 1 & 81.625 & 85.375 \\
Sub 2 & 82.2 & 85.25 \\
Sub 3 & 82.125 & 85.55 \\
Sub 4 & 81.725 & 84.75 \\
Sub 5 & 81.5 & 84.9 \\
Final average & 81.835 & 85.165 \\
\hline \hline
\end{tabular}

After segmentation of images, morphological operations use to obtain individual lung and to remove unnecessary parts. Morphology is a technique that works on processing images based on shapes. A structuring element is a shape mask used in the basic morphological operations applies a structuring element to an input image, creating an output image of the same size. The basic morphological operations are dilation and erosion .The different morphological operators used are below:

- Dilation : The process of dilation is the ability $t$ o add pixels to the boundaries of objects whic $h$ means it's a process of intensification or gro wth of objects in the binary image

- Erosion: Erosion removes pixels on object boundaries in an image.

- Opening: Opening can be used for eliminating protrusions, breaking necks and smoothening contours.

- Closing: Closing can be used for fusing narrow breaks and long thin gulfs, eliminating small holes, filling gaps in the contour and smoothing contours.

\section{D.Feature Extraction:}

It predicts the probability of the presence of cancer therefore, it's an important stage .it uses 
algorithms to detect or isolate the shape or feature of an image. The following method is used:

\section{Binarization :}

It's a process of altering the color of the pixels to black and white. After counting the number of black and white pixels it compares it to a threshold value it shows if the image is normal or abnormal.

\section{RESULTS}

The original image of CT natural lung is shown in Fig. 2 (a), and the abnormal CT is shown in Fig. 2 (b)

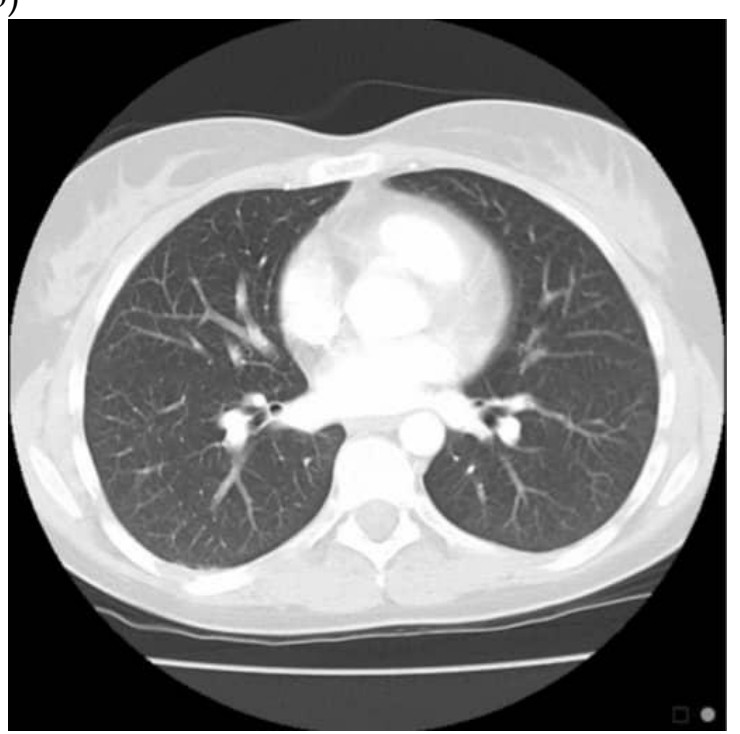

Fig. 2(a) image of CT normal lung

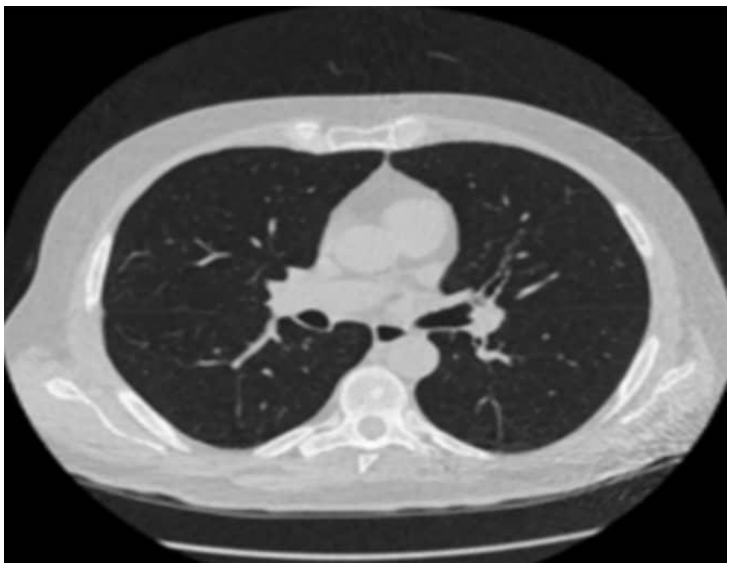

Fig. 2(b) image of CT abnormal lung

Fig3 describes the preprocessed image when the mean and median filter and used for reducing noise, Fig. 3(a) shows enhancement for a normal image and Fig. 3(b) shows enhancement for an abnormal image.
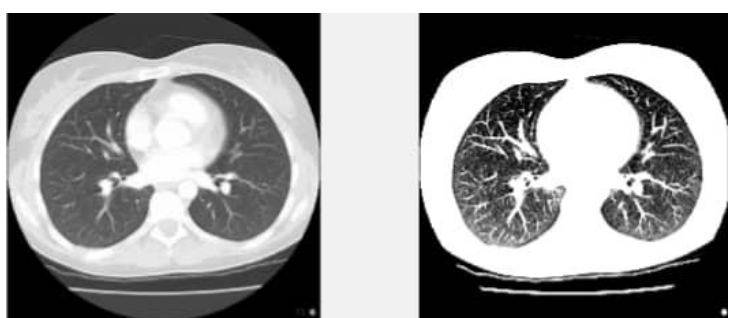

Fig. 3(a) enhanced normal image
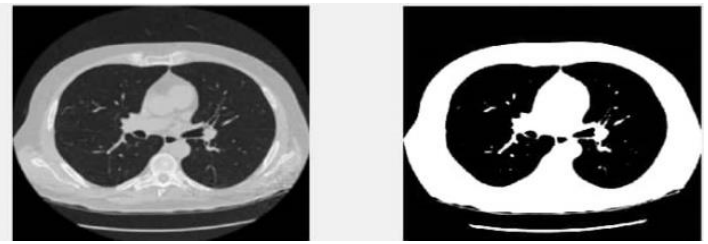

Fig. 3(b) enhanced abnormal image

After that we concluded that there is a cancerous tumor or not as shown in Fig. 4, Fig. 4(a) is the result of normal lung and Fig. 4(b) is the result of cancerous lung

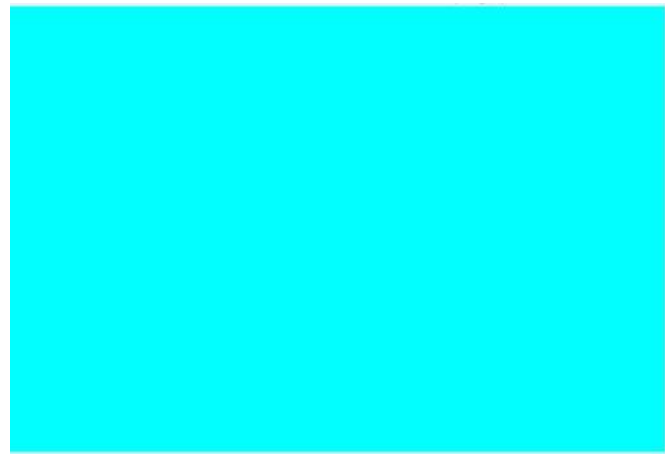

Fig. 4(a) the resulted image indicates normality

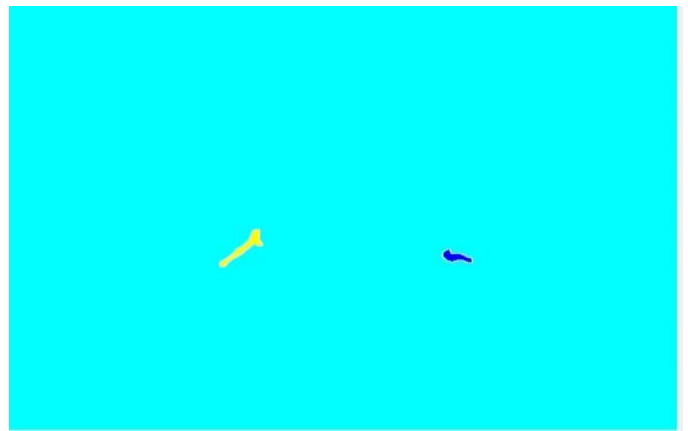

Fig. 4(b) the resulted image indicates abnormality

Finally, we segment the image by using watershed technique fig. 5 describe results after using this technique, fig.5(a) shows segmentation for a normal lung and fig.5(b) shows segmentation for a abnormal lung.

To discriminate between the healthy lung and the infected lung we pay attention to the color of the image. If it shows one color (Blue) this means the lung is normal and if it shows multiple colors this means that the lung contains a tumor. 


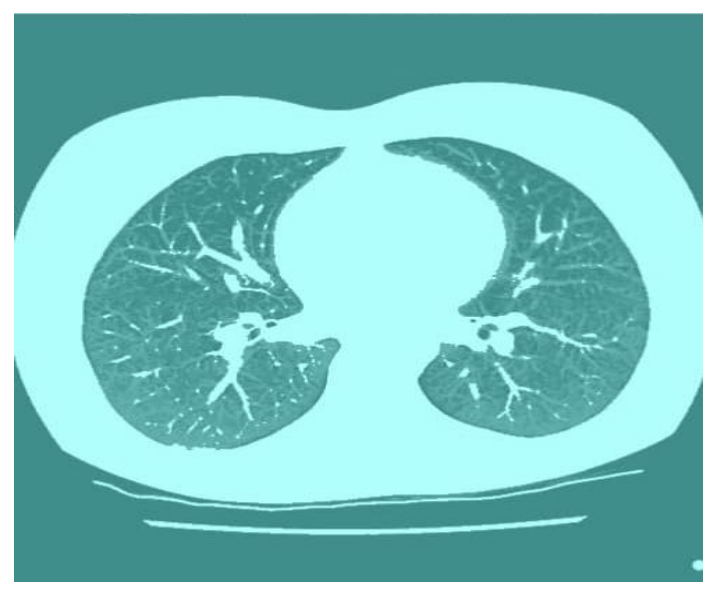

Fig. 5(a) watershed on normal lung

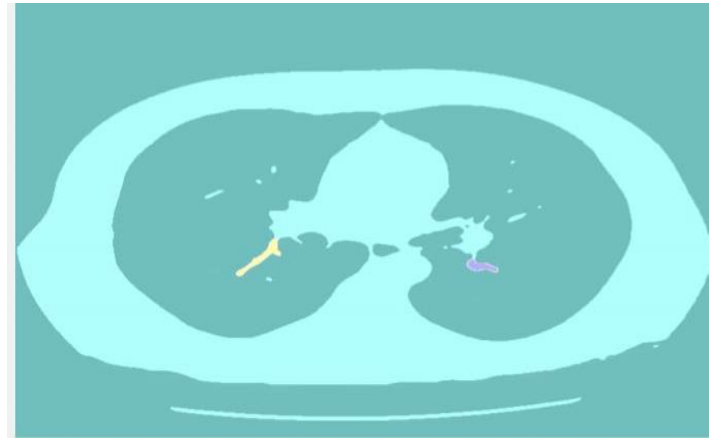

Fig.5(b)wa tershed on abnormal lung

\section{CONCLUSION}

Lung cancer is the most deadly type of cancer, which can be detected in its early stages by detecting the nodules of the lungs. In this paper, a diagnosis of the disease is obtained by detecting the lung's nodules by improving the image and then dividing the image taken into the infected vision.
The features of the image are extracted to show us whether the image contains a tumor or not. This technique helps doctors and radiologists to take the correct and accurate decision in a short time compared to other methods and by providing the appropriate information, so this method is considered easy and inexpensive for others.

\section{REFERENCES}

[1] R. Nall, MSN, CRNA, ( November 16, 2018),Medicalnewstoday available :https://www.medicalnewstoday.com/articles/323701.php [2] E. Downward ,(March 2019), How Common Is Lung Cancer?, available :https://lungcancer.net/basics/statistics/

[3] 1300 N. 17th Street, Suite 1847 , Digital Imaging and Communications in Medicine (DICOM), Rosslyn: Virginia 22209 USA, September 6, 2002 , part1, pp.11.

[4] A.KRISHAN, "Evaluation of Gabor filter parameters for image enhancement and segmentation", M. S. thesis, Dept. of Electrical and Instrumentation Eng, Tharap univ, Patiala, punjap, 2009.

[5] Z. R. Hussein, R. W. Rahmat, "Pre-processing Importance for Extraction Contours from Noisy Echocardio graphic Images", International Journal of Computer Science and Network Security, Image Processing, vol.9, No.3, March 2009.

[6] M.Singh , P.K.Gupta, V.Tyagi, J.Flusser and T.Oren, "Advances in Computing and Data Science Part Two", ICACDS 2018, India :Dehradun, 2018 , p 546.

[7] B. Abdillah,. Bustamam, and D. Sarwinda,"Image Processing Based Detection of lung cancer on CT Scan Images",Faculty of Mathematics and Science, University of Indonesia, December 24,2018.

[8] Mokhled S. AL- TARAWNEH,"Lung Cancer Detection Using Image Processing Techniques", Computer Engineering Department, Faculty of Engineering, Mutah University, . August 2012. 